\title{
Dynamic stability training improves standing balance control in neuropathic patients with type 2 diabetes
}

\author{
Hoda Salsabili, MS; ${ }^{*}$ Farid Bahrpeyma, PhD; ${ }^{1}$ Bijan Forogh, MD; ${ }^{2}$ Sanaz Rajabali, MS $^{\mathbf{3}}$ \\ ${ }^{1}$ Department of Physical Therapy, Tarbiat Modares University, Tehran, Iran; ${ }^{2}$ Department of Physical Medicine, \\ Tehran University of Medical Sciences, Tehran, Iran; ${ }^{3}$ Department of Biomedical Engineering, Islamic Azad Univer- \\ sity, Tehran, Iran
}

\begin{abstract}
Patients with type 2 diabetic neuropathy (DN) are at an increased risk of falls due to the decreased accurate proprioceptive feedbacks. Effective balance training should treat context-specific instabilities of postural control of patients with DN. For this purpose, evaluations and balance training were designed with a 3-week baseline with evaluation after 3 weeks, followed by training over 3 weeks with reevaluation. We acquired control scores for standing balance from the Biodex stability system and fluctuations of the center of pressure. We performed repeated measure analysis of variance to test mean differences in three sessions of assessments. In addition, we compared mean differences of each pair of sessions with the least significant difference test. We used the paired $t$-test to compare the pure effects of trainings. Our investigation showed that the results of Biodex stability scores and force platform medial-lateral sway measurements improved statistically. Significant higher open-eyes median and mean frequency values of postural sway in the medial-lateral direction indicated that balance training allowed patients to develop control over the degree of freedom at the hip joint. In conclusion, training that compensates for disordered balance indicated by subclinical constraints with respect to the guidance effect of external visual feedback improves standing postural control in patients with type $2 \mathrm{DN}$.
\end{abstract}

Clinical Trial Registration: Clinical Trial Registration: UMIN-CTR Search Clinical Trials, UMIN000004485, "Dynamic stability training can promote quiet standing control in neuropathic patients with type 2 diabetes"; http:// www.umin.ac.jp/ctr/index.htm.
Key words: balance training, context-specific instabilities, COP, diabetic neuropathy, external visual feedback, force platform, postural control, postural stability scores, postural sway, proprioception, rehabilitation.

\section{INTRODUCTION}

Patients with type 2 diabetic neuropathy (DN) are at an increased risk of falls. This increased risk is presumably due to the well-documented balance problems attributed to neuropathy [1] and sensory ataxia, which is the lack of accurate proprioceptive feedback [2]. Sources of instability in patients with type $2 \mathrm{DN}$ include the loss or reduction of peripheral sensory information in the feet [3-4], the inability of the central nervous system (CNS)

Abbreviations: $\mathrm{ANOVA}=$ analysis of variance, $\mathrm{AP}=$ anteriorposterior, APSI = AP stability index, BMI = body mass index, BSS = Biodex stability system, CNS = central nervous system, $\mathrm{COM}=$ center of mass, $\mathrm{COP}=$ center of pressure, $\mathrm{DN}=$ diabetic neuropathy, LSD = least significant difference, $\mathrm{ML}=$ medial-lateral, MLSI $=$ ML stability index, OSI $=$ overall stability index, SD = standard deviation.

*Address all correspondence to Hoda Salsabili, MS; Department of Physical Therapy, Tarbiat Modares University, Jalal Al Ahmad Street, Gisha Bridge, Tehran, 1458845978, Islamic Republic of Iran; 0098-021-77927852; fax: 0098-021-66018515. Email: h.salsabili@gmail.com DOI:10.1682/JRRD.2010.08.0160 
to appropriately integrate available postural control information [2,4-5], and a switch from an ankle-based to a hip-based balance strategy [6-10]. In addition, an increase in the use of vestibular information and dependence on visual information [11] alter the style of postural control in patients with DN.

Stability can be defined as the sensitivity of a dynamic system to perturbations, and local stability is the sensitivity of the system to internal perturbations, such as natural fluctuations (e.g., changing muscle activity in response to gravity), that occur during posture [12]. The effects of these natural fluctuations were examined for evaluating different measures of postural sway [13]. Reported studies on patients with diabetes with neuropathic postural sway suggest relative deficits in their ability to maintain posture and indicate greater instability than that observed in patients without diabetes [7] and positive relation between postural sway and falling [14]. Also, studies of postural instability in patients with DN have indicated higher scores for postural stability indexes [15], speed, and area of sway [2,16]; higher center of pressure (COP) ranges [2]; higher root-mean-square values of the COP-center of mass (COM) variable [17] (time domain); and greater increase of sway power within medium-high frequencies $(0.5-1.0 \mathrm{~Hz})$ [18] in postural sway variables that indicate lower stability control.

Understanding and properly performing stability and balance training in patients require complete knowledge of the sources of patients' postural problems. Postural control systems contain a complex organization that controls the orientation and the equilibrium of the body during upright stance. Additionally, control of posture involves different underlying physiological systems and depends on six contexts: (1) biomechanical task constraints, (2) movement strategies, (3) sensory strategies, (4) orientation in space, (5) control of dynamics, and (6) cognitive processing [19-20]. Sources of sensory afferents that seem to contribute to postural control include (1) proprioception, (2) vestibular, and (3) visual systems [3]. Pathological or subclinical constraints in patients with DN are due to distinct, context-specific instabilities such as movement [4] and sensory (somatosensory) strategies [20]. Reactive movement strategies help patients develop coordinated multijoint movement, and sensory strategies help patients to select appropriate sensory information for postural control [21]. Ankle and hip reactive movement strategies can be used to return the body to equilibrium and keep the feet in place. When persons stand on a firm surface, ankle strategy maintains balance with small movements of sway, like an inverted pendulum. Hip strategy controls the quick and narrow movements of the COM when a person stands on a narrow or compliant surface that allows inadequate ankle torque [4]. Retraining reactive movement strategies involves exposing the patients to external perturbations that vary in direction, speed, and amplitude [21].

While some well-performed studies have described the effects of balance training on patients with DN $[13,22-24]$, few investigations have attempted to mitigate the context-specific postural instability problem in this population [24-25]. Some studies, however, have reported on the correlation between balance training and visual external feedback. This training was used to improve the dynamic balance and sensory integration capabilities of elderly adults with a history of falls [26] and was helpful for frail older women [27] and elderly women living in residential care [28]. It also has been preferred to home-based balance training [29]. Furthermore, static-standing steadiness can be trained effectively through weight-bearing biofeedback [30], and visually guided weight-shifting training can improve standing balance control of static balance in elderly women [31]. Because patients with DN are often older and have instability symptoms similar to those of elderly people [32], we suspected that a balance-training program with external visual biofeedback developed for the elderly might also improve balance in patients with DN.

To rehabilitate balance effectively in patients with $\mathrm{DN}$, one must consider the subclinical constraints imposed by altered somatosensation and disordered balance relative to normal CNS strategies. In light of these concerns, a physical therapy team must encourage patients with DN to compensate by using the somatosensory system and ankle proprioception [4]. For instance, training with respect to sensory, ankle, and hip strategies appears to be useful for improving patients with DN impaired somatosensory subclinical constraints. In this way, the Biodex stability system (BSS) (Biodex 945-302, Biodex Medical Systems Inc; Shirley, New York) presents a system that can provide specific ankle and hip postural strategy training with external visual biofeedback as a guide to improve the impaired subclinical constraints of patients with DN [26].

In the present study, we hypothesized that effective balance training should treat context-specific instabilities of postural control of patients with DN. In other words, 
our balance training methods borrowed from reactive movement strategies and sensory strategies to provoke somatosensory information regarding the guiding contributions of external visual biofeedback. For this purpose, the physical therapy team asked the patients with DN in this study to control perturbations due to the instability and gravity effects of an unstable platform based on targets providing external visual biofeedback for balance training. These targets cued slow and small movements to provoke ankle strategy, and they indicated fast and large movements to activate hip strategy. We planned to observe the use of these strategies when patients with DN attempted to tilt the unstable platform following guidance target cues and in the context of coincidental gravity perturbations induced by the unstable platform that influenced body equilibrium.

\section{MATERIALS AND METHODS}

\section{Participants}

A group of 19 neuropathic patients with type 2 diabetes was recruited for a quasi-experimental time-series study. A physical medicine physician assessed neuropathies of patients using nerve conduction velocity tests (Table 1). Patient inclusion criteria were patient age (between 40 and 70 years old), duration of time suffering from controlled type 2 diabetes ( $>5$ years), patient fasting blood sugar test result ( $>110 \mathrm{mg} / \mathrm{dL}$ ), Valk neuropathy score ( $>2)$, and Snellen chart score (>16/20). Participants were excluded from this study if they had retinopathy, scars under their feet, hypotension, autonomic neuropathy, or any orthopedic or neurological impairment related to balance performance. They were also excluded if they had prior experience with balance training using the BSS, force platform, and the task.

Table 1.

Patients' ( $N=19)$ baseline descriptive and demographic characteristics.

\begin{tabular}{lc}
\hline \multicolumn{1}{c}{ Descriptive Information } & Patients \\
\hline Sex & 12 women and 7 men \\
Age $(\mathrm{yr})$ & $56.00 \pm 8.96$ \\
Height $(\mathrm{cm})$ & $164.52 \pm 5.09$ \\
Weight $(\mathrm{kg})$ & $76.52 \pm 12.87$ \\
Body Mass Index $\left(\mathrm{kg} / \mathrm{m}^{2}\right)$ & $28.33 \pm 4.65$ \\
Diabetic History $(\mathrm{yr})$ & $11.84 \pm 6.93$ \\
\hline Note: Values are mean \pm standard deviation. & \\
\hline
\end{tabular}

\section{Assessments and Measurements}

This study included a 3-week baseline, followed with an evaluation (time effect) by training over 3 weeks with reevaluation (training effect) (Figure 1). The first evaluation included descriptive information such as age, sex, height, weight, body mass index (BMI), Valk score, diabetic history, and postural strategy assessments with BSS scores and force platform assessments. After the first 3-week period, patients were evaluated for the second time with Valk score and postural strategy assessments. The third evaluation again included Valk score and postural strategy assessments after the patients had been trained by Biodex balance exercises in the second 3-week period.

Neuropathy was assessed by a nerve-conduction velocity test. For this purpose, the amplitude and latency of the sensory (sural) and motor (proneal and tibial) nerves were recorded. The motor or sensory nerve conduction velocity of $39 \mathrm{~m} / \mathrm{s}$ was adopted as the lower limit (cutoff value: below average $=2$ standard deviation [SD] of the normal range) [16,33].

The Valk polyneuropathy score assessed the severity of neuropathy based on the frequency of symptom occurrence. This instrument is a 10-item sensory polyneuropathy score that has two different subdimensions to evaluate the sensory alteration and neuropathic pain that comprise part of the diabetic symptoms checklist-type 2 .

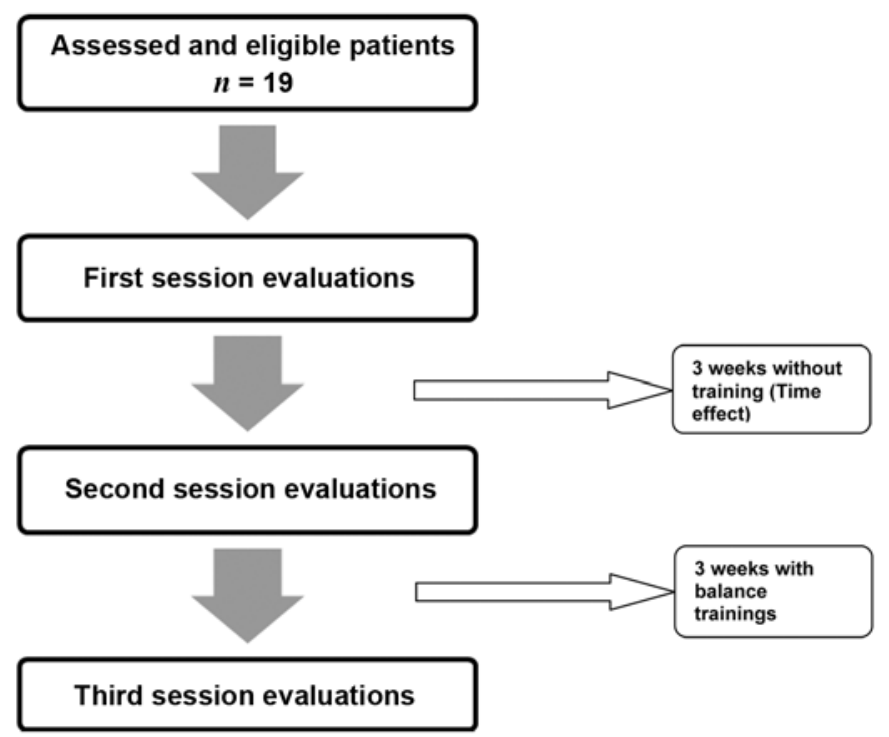

Figure 1.

Flow chart for method of recruiting and assessing patients for balance training. 
We reported the construct validity between symptom severity and nerve fiber function (Pearson correlations) and confirmed the reproducibility of this instrument by test-retest correlation coefficients of the assessments of the severity of sensory alteration complaints and neuropathic pain (0.89 and 0.85) [34].

Standing balance control scores were acquired from BSS (Figure 2). The Biodex balance system uses a circular platform that is free to move about the anteriorposterior (AP) and medial-lateral (ML) directions simultaneously. Also, one can change the stability of the platform by varying the resistance force applied to the platform by underside springs. The springs' forces are adjustable to preset resistance levels that determine the difficulty levels of BSS. This device measures the degree of tilt about each axis of ML and AP during dynamic conditions by two
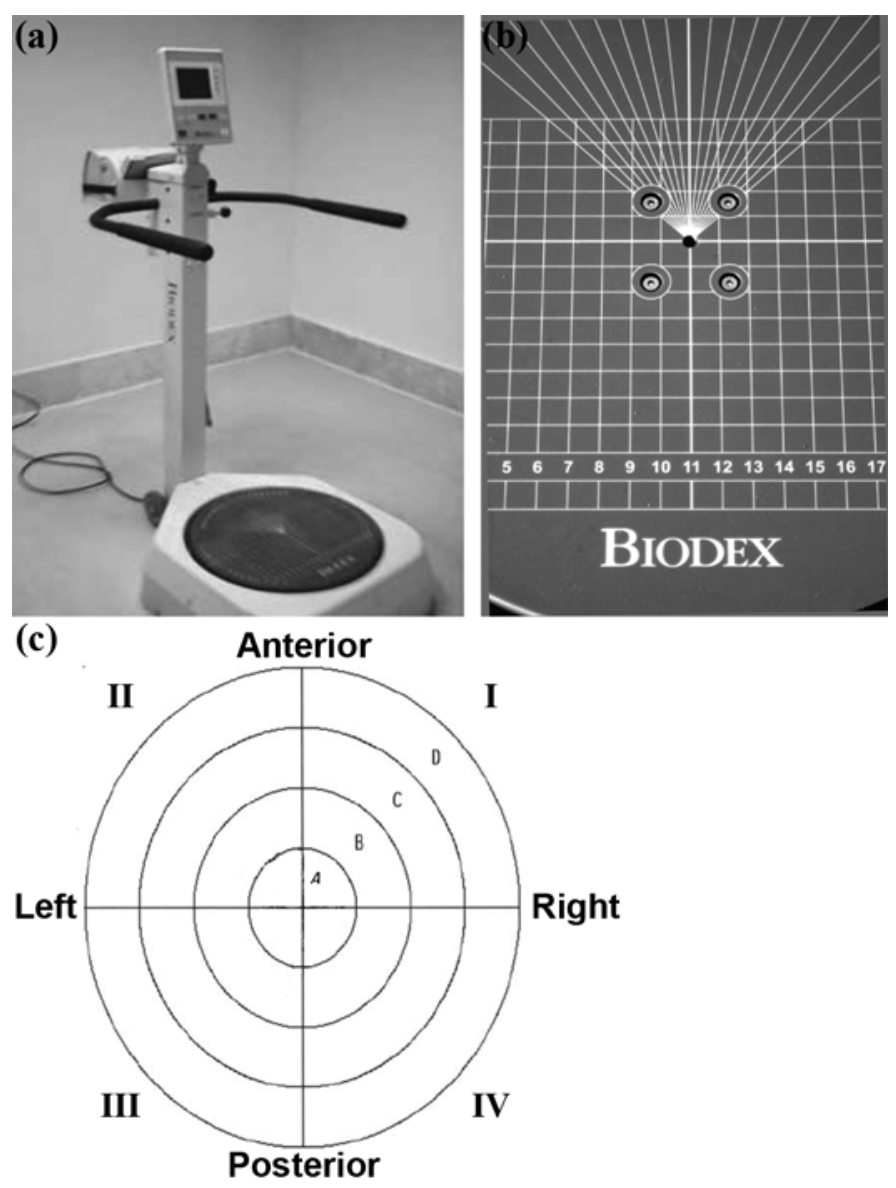

Figure 2.

(a) Biodex stability system, (b) stability platform, and (c) external biofeedback screen for specific ankle and hip postural strategy training with external visual biofeedback. under-platform potentiometers that record tilting. Its movable balance platform provides up to $20^{\circ}$ of surface tilt in a $360^{\circ}$ range of motion. The platform is connected to computer software (Biodex, Version 3.1; Biodex Medical Systems) that enables the device to be used objectively for assessing balance. The BSS provides information more specifically on ankle joint movements. The BSS calculates overall stability index (OSI) from the degrees of tilt about the AP and ML axes and separately calculates ML stability index (MLSI) and AP stability index (APSI) (a high score in the indexes indicates poor balance [35]) by

$$
\text { OSI }=\sqrt{\frac{\sum(0-Y)^{2}+\sum(0-X)^{2}}{\text { samples }}}
$$

$$
\begin{gathered}
\text { APSI }=\sqrt{\frac{\sum(0-Y)^{2}}{\text { samples }}}, \\
\text { MLSI }=\sqrt{\frac{\sum(0-X)^{2}}{\text { samples }}},
\end{gathered}
$$

where $Y=$ the tilt angle of tilt board in the ML direction and $X=$ the tilt angle of tilt board in the AP direction.

At first, the therapy team familiarized the patients with the device. For the familiarization trial, patients stood on the platform with their hands at their sides while maintaining a comfortable knee angle. Then in this position, the stability platform was unlocked to allow motion. The team then instructed the patients to adjust feet positions until they found a position at which they were able to maintain a moving point in the center or near the center of the circles, with the difficulty level of 8 (the balance test is most difficult when the platform provides the least resistance to tilting and is therefore the least stable). The difficulty level of 8 is the easiest level that has the most resistance to the unstable platform. Next, the platform was locked and the feet placement was recorded and saved for assessing trials in all testing sessions. To achieve indexes from each patient, we asked them to complete three trials with visual biofeedback and three trials without visual biofeedback. Each trial lasted $30 \mathrm{~s}$, with the difficulty level of 8, and followed the same method as the familiarization trial [35]. In the study by Schmitz and Arnold, they reported an intratester reliability of 0.80 for the APSI, 0.43 for the MLSI, 
and 0.82 for the OSI by using a decreasing stability protocol from level 8 to level $1>30 \mathrm{~s}$ [36].

Force platform measurements (Kistler 9286BA; Winterthur, Switzerland) were used for evaluating the quantification of fluctuations in the COP. The COP is the single point location of the ground reaction force vector [37]. The reliability of COP measures was studied by Lin et al., who reported intraclass correlation values for AP and ML direction for within-day (0.57 and 0.77) and between-day ( 0.57 and 0.56 ) measurements for median power frequency [38]. To assess patients in an upright stance, we instructed the patients to stand as still as possible on a force platform. We asked them to place their heels next to each other, making a $30^{\circ}$ angle between the medial borders of their feet and then to look straight ahead to the marker $1.5 \mathrm{~m}$ away while comfortably hanging their arms at their sides. Six successive trials were conducted consisting of three trials with open eyes and three trials with closed eyes. Each trial lasted $30 \mathrm{~s}$, and the sampling rate was $50 \mathrm{~Hz}$. The rest period between each trial was 2 minutes. The signals were filtered with a zero-lag Butterworth low-pass filter at $5 \mathrm{~Hz}$. For the present study, we reported the mean and median of frequency $(f)$ of COP signals (higher mean and median frequencies indicate better balance performance [12]) according to

$$
\begin{aligned}
& f \text { mean }_{\mathrm{AP}}=\frac{\sum_{i=1}^{i=n \mathrm{fft} / 2} f_{i} \mathrm{PSD}(x)_{i}}{\sum_{i=1}^{i=\mathrm{ft} / 2} \operatorname{PSD}(x)_{i}}, \\
& f \text { mean }_{\mathrm{ML}}=\frac{\sum_{i=1}^{i=n \mathrm{fft} / 2} f_{i}{ }^{*} \mathrm{PSD}(y)_{i}}{\sum_{i=1}^{i=\mathrm{fft} / 2} \operatorname{PSD}(y)_{i}}, \\
& f \operatorname{med}_{\mathrm{AP}}=\frac{\sum_{i=1}^{f=\operatorname{med}} \operatorname{PSD} f(x)}{\sum_{i=1}^{i=n \text { fft } / 2} \operatorname{PSD} f(x)}=\frac{1}{2}, \\
& f \operatorname{med}_{\mathrm{ML}}=\frac{\sum_{i=1}^{f=\operatorname{med}} \operatorname{PSD} f(y)}{\sum_{i=1}^{i=n t f t / 2} \operatorname{PSD} f(y)}=\frac{1}{2},
\end{aligned}
$$

where med = median, $n=$ the total number of samples, $y=$ the coordinates of COP in the ML direction, $x=$ coordinates of COP in the AP direction, $\mathrm{fft}=$ the fast Fourier transformation, and PSD = power spectral density.
In this study, we included force platform evaluations to avoid training and learning effect of BSS. Also, almost all BSS balance training practices (except standing practice with covered biofeedback) had different approaches from testing. Additionally, with respect to the progressions toward less stable levels in every session and practices, we could claim that they were completely different.

\section{Task Trainings}

We conducted standing balance trainings by using BSS for 10 sessions in 3 weeks, every session lasting 30 minutes with a 1-day interval and the same standing method and feet placement of the familiarization trial. At the beginning of each session, we tested patients' blood sugar level using an ACCU-CHEK glucometer (Roche Diagnostics; Indianapolis, Indiana) to be over $7.8 \mathrm{mmol} / \mathrm{L}$ to control hyper- or hypoglycemia. Trainings consisted of two limits of stability, three weight shiftings with visualexternal biofeedback practices, and one standing practice with covered biofeedback. All trainings progressed from easy to difficult, with the stability level decreasing through sessions. Investigators adjusted level progressions according to the patients' ability and assigned limits (Table 2).

Throughout the study, investigators allowed the patients to find the best strategy for standing on the BSS platform, which perturbed patients by means of gravity and unstable platform. Although gravity is constant, equilibrium is unstable and small fluctuations are seen in balance measures [39]. Despite the effect of gravity, the unstable platform would use sensory and reactive movement strategies $[21,40]$.

\section{Sample Size Calculation}

According to the studies by Biodex Corporation, OSI for a nondisabled age-matched group was $2.15 \pm 0.75$ (http://www.biodex.com/rehab/balance/

balance_440feat.htm\#description) compared with $2.53 \pm 0.85$ in patients with DN in our study. With respect to improvement of patients with DN in their OSI from 2.53 to 1.58 after balance trainings, the effect size was 0.7 . Therefore, assuming the effect size of 0.5 (medium) to have a larger group of study, a significant $\alpha=0.05$, and a statistical nominal power of 0.88 , we needed 19 participants to test these relationships as indicated in Cohen's table [41].

\section{Data Analyses}

We analyzed the patients' normal distribution baseline means with the Kolmogorov-Smirnov test. We performed repeated measure analysis of variance (ANOVA) 
JRRD, Volume 48, Number 7, 2011

Table 2.

Pattern and context of training, feet placement, time, and promotion of difficulty levels through training sessions.

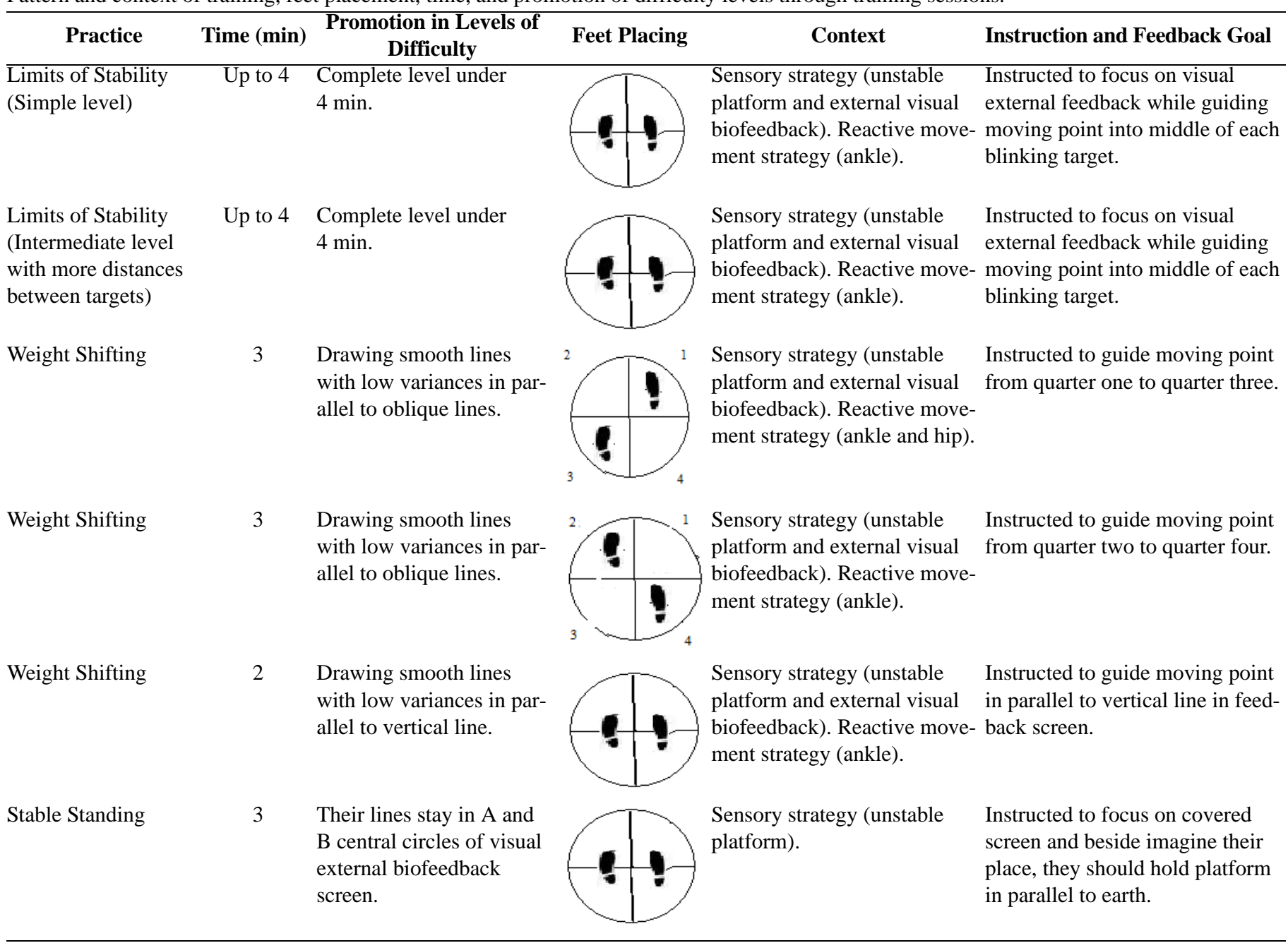

to test mean differences in three sessions of assessments. Mean differences of each pair of sessions were compared with the least significant difference (LSD) test. We used the paired $t$-test to compare the pure effects of trainings (Figure 3), which included the differences between paired sessions of trials with and without biofeedback conditions with BSS assessments as well as between open or closed eyes conditions in force platform assessments. SPSS version 15 (SPSS Inc; Chicago, Illinois) was used for all statistical analyses.

\section{RESULTS}

All patients performed training without any complications and agreed to the use of their training data. Kolmog-
orov-Smirnov analysis of normality showed that data distribution assessments of all three sessions were normal.

\section{Valk Neuropathy Severity Scores}

Mean \pm SD of the scores of three sessions (Table 3) showed reduction in the Valk scores of the third session. Repeated measure ANOVA indicated that within-subject effects failed to reach significance $(p=0.07)$.

\section{Biodex Stability System Indexes}

The mean BSS indexes and their SDs from three sessions (Table 3) showed reductions for OSI in both with or without visual feedback conditions ( 2.53 to $1.58,3.67$ to 2.60), MLSI (1.57 to $1.21,1.96$ to 1.57$)$, and APSI (2.10 to $1.55,3.15$ to 2.15$)$ in the third session of assessments. In the first step, repeated measure ANOVA indicated that 


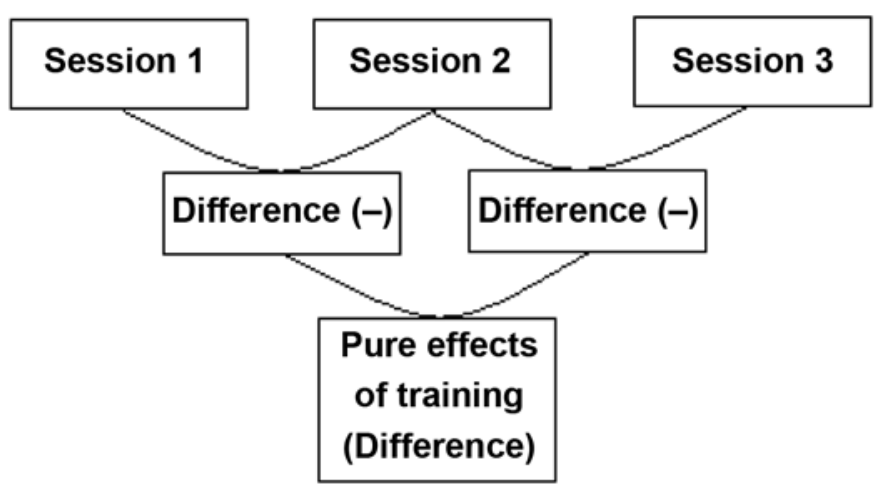

Figure 3.

Differences of pure effects of training.

within-subject effects reached significant differences for OSI in both with and without visual feedback conditions $(p<0.001, p<0.001)$, MLSI $(p<0.001, p=0.02)$, and APSI $(p<0.001, p<0.001)$. In the second step, the LSD test indicated significant differences with visual feedback for OSI, MLSI, and APSI between the second and third assessment sessions ( $p=0.001, p<0.001, p=0.003)$ and first and third sessions ( $p<0.001, p<0.001, p<0.001)$ and failed to reach any significant differences between the first and second assessment sessions ( $p=0.06, p=0.95$, $p=0.38$ ). The LSD test also indicated significant differences without visual feedback for OSI, MLSI, and APSI between the second and third assessment sessions $(p<$ 0.001, $p<0.001, p=0.05)$ and the first and third ( $p=$ $0.001, p=0.006, p=0.001$ ) and failed to reach any significant differences between the first and second assessment sessions ( $p=0.97, p=0.05, p=0.45$ ). Paired t-test analysis showed no significant differences between the pure effects of trainings in BSS assessments with biofeedback and without biofeedback conditions for OSI, MLSI, and APSI ( $p=0.21, p=0.20, p=0.78)$.

\section{Force Platform Variables}

The mean \pm SD of force platform variables increased in the mean and median of frequency of COP signals for either open or closed eyes conditions in the third session of assessments (Table 3). Repeated measure ANOVA indicated that within-subject effects of open eyes conditions reached significant differences for the ML mean and median of frequency of COP signals ( $p=0.005, p=0.01$ ) but failed to reach any significant differences for the AP mean and median of frequency ( $p=0.18, p=0.21)$. This

Table 3.

Mean \pm standard deviation of Valk scores, Biodex stability system indexes, and force platform variables in three sessions.

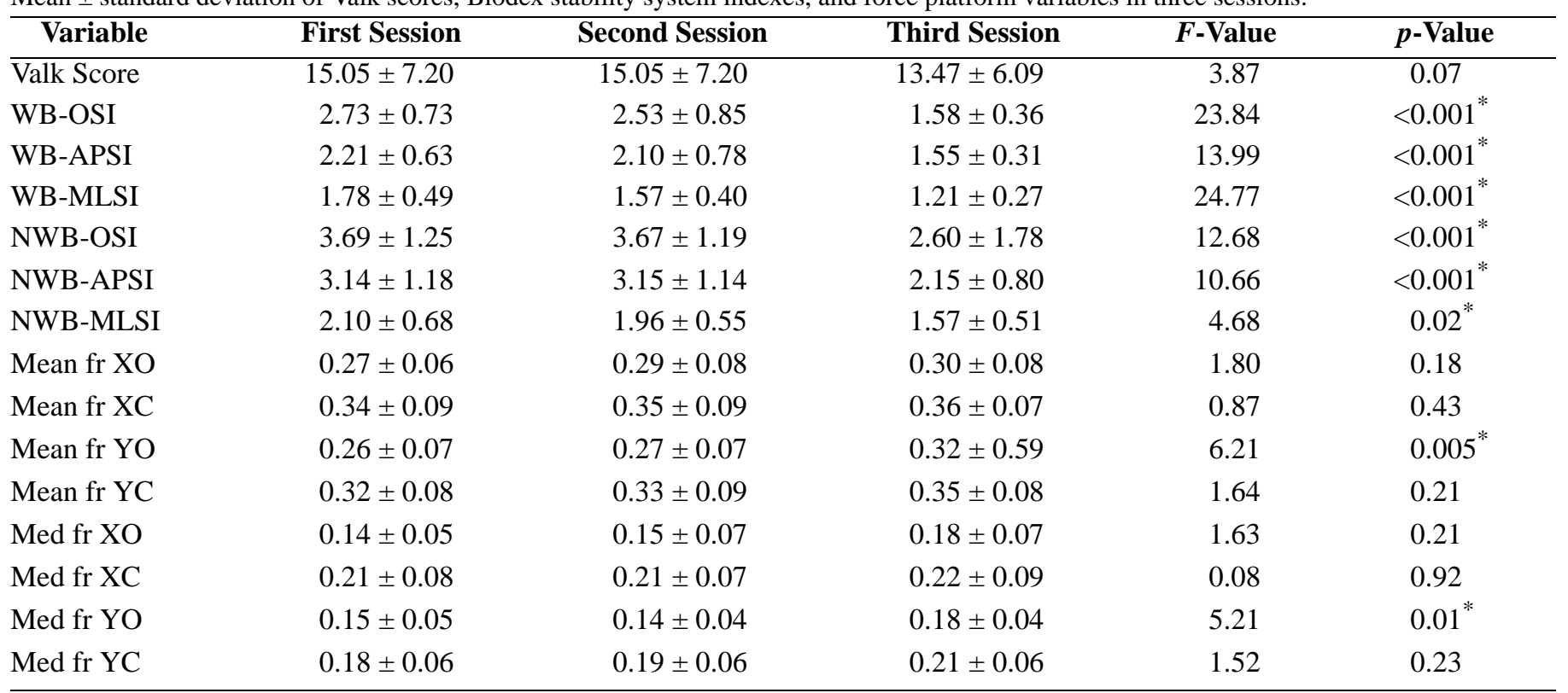

*Statistically significant differences between sessions $(p<0.05)$.

APSI = anterior-posterior stability index, Mean $\mathrm{fr}=$ mean frequency $(\mathrm{Hz}), \mathrm{Med} \mathrm{fr}=$ median frequency $(\mathrm{Hz}), \mathrm{MLSI}=$ medial-lateral stability index, $\mathrm{NWB}=$ without biofeedback tests, OSI = overall stability index, $\mathrm{WB}=$ with biofeedback tests, $\mathrm{XC}=$ anterior-posterior direction with closed eyes, $\mathrm{XO}=$ anterior-posterior direction with open eyes, YC = medial-lateral direction with closed eyes, $\mathrm{YO}=$ medial-lateral direction with open eyes. 
analysis did not reveal differences in the closed eyes condition for the AP mean and median of frequency ( $p=$ $0.43, p=0.92$ ) or the ML mean and median of frequency ( $p=0.21, p=0.23$ ). The LSD test indicated significant differences for the open eyes: ML mean and the median of frequency of COP signals between the second and third paired sessions ( $p=0.02, p=0.005$ ), and the first to third ( $p=0.009, p=0.04)$ but failed to reach any significant differences between the first and second paired sessions ( $p=0.63, p=0.26$ ). Paired $t$-test analysis showed no significant differences between the pure effects of trainings in mean and median of frequency analysis between the assessment conditions of closed and open eyes in the AP $(p=0.88, p=0.91)$ and ML directions $(p=0.62, p=0.39)$.

\section{DISCUSSION}

In the present study, we hypothesized that effective balance trainings should treat context-specific instabilities of postural control of patients with DN by placing more emphasis on somatosensory information in balance training. By means of these trainings, our balance training methods borrowed from reactive movement strategies and sensory strategies for evoking somatosensory information regarding the guiding contributions of external visual biofeedback. In this study, patients with DN improved their BSS assessment scores and force platform evaluations with open eyes in the ML sway direction. Importantly, the Valk severity score did not show any significant difference in the severity of DN in the process of trainings and assessments, indicating that the better balance performance in patients with DN was not the consequence of any changes in the severity of neuropathy.

Clinical constraints in patients with DN are due to distinct, context-specific instabilities such as movement strategies (hip and ankle) [4] and sensory strategies (specifically somatosensory) [20]. Movement studies have shown that ankle strategy is responsible for slow and small movements of the COM at low velocities but that hip strategy controls larger movements of the COM at higher velocities [21]. Investigation of the sources of instability in patients with DN has shown the need to rehabilitate their somatosensory system and ankle movement strategy [4]. In our study, we conducted retraining reactive balance control in patients with DN by instructing them to control perturbations due to gravity and plat- form instability [21]. For instance, training sessions composed of small and large self-initiated movements of a platform were guided by external visual biofeedback that was noted to recruit proprioceptive inputs to process sensory strategies during training sessions. While standing on an unstable platform, patients with DN countered with gravity perturbations that influenced body equilibrium by using the strategies based on the targets in the feedback screen to guide the unstable platform.

We added force platform analysis to confirm better balance performance of patients with DN in situations distinct from biofeedback training. The studies with the force platform indicated that stiffness control in the AP direction of quiet standing is located at the plantar flexors and in the ML direction is centered in the hip abductor and adductors. Also, in the side-by-side stance, the ankle muscles control the AP balance while hip muscles control the ML balance [42]. Studies of patients with DN demonstrate that they are less stable in the ML direction of postural sway parameters [10]. The biomechanical and sensory problems of these patients at the ankle level lead to compensation with the more readily available postural control information at the hip level. This control information increases patient sensitivity to information in the ML axis [4]. Furthermore, several studies have introduced lateral instability as a marker of impaired balance control in older persons [43] and in patients with DN [44]. This instability implies that reliance on the hip joint does not improve postural control but can increase the amount of sway with lower mean frequency during standing in the ML direction [45]. The same study showed that a higher frequency of COP signals from the force platform represents better postural control [45]. Thus, the significant higher open-eyes median and mean frequency values of postural sway in the ML direction that our subjects exhibited after training indicate better balance performance and showed that balance training allowed patients to regain control of a degree of freedom of their hip joints. These results are consistent with the findings of Nagy et al., who studied elderly subjects who participated in combined biweekly training for 8 weeks in 4 min sessions [46]. Their exercise program included combinations of lowerlimb strength and flexibility exercises, static and dynamic balance exercises, and walking as an aerobic activity. Their balance confidence and control of ML balance in response to the 8 weeks training improved, and the higher ML direction frequency power exhibited after the training may indicate better balance performance [46]. 
The statistically improved BSS results we found are consistent with results of other studies that introduced balance training as a useful method for improving balance. Rose and Clark implemented an 8-week balance intervention with computerized biofeedback training for older fallers and measured the dynamic postural control and sensory organization before and after balance training [26]. The dynamic balance and sensory integration capabilities of the intervention group improved significantly, indicating that rehabilitation programs could concurrently and significantly improve the control of bodily orientation of older adults with a previous history of falls in both static and dynamic action environments [26]. Sihvonen et al. examined the effects of 4-week visual-feedback-based balance training on the fall incidence during a 1-year follow-up among frail older women living in residential care [27-28]. They concluded that visual feedback-based balance training has been shown to be a promising method for fall prevention among frail older women [2728]. Another investigation that confirmed the effectiveness of BSS balance training was the study of Lindemann et al., who compared the effectiveness of computerassisted balance training with a home-based exercise program in nondisabled elderly subjects [29]. The study showed that computer-assisted balance training, even if focused on one motor skill, was more effective than a home-based exercise program [29]. Nardone et al. confirmed that balance rehabilitation with either specific physical exercises or a powered platform is effective in patients with balance disorders of neuropathic origin; thus, they contribute to increasing stability and potentially decreasing the risk of falling in patients with neuropathy [47]. Also, Nardone et al. showed that sinusoidal perturbation during a dynamic task provides larger sensory inputs and may supplement lower-limb sensitivity [48]. The results of Mattacola and Lloyd revealed that manual muscle strengthening and proprioception training for the plantar flexor, dorsiflexor, inversion, and eversion muscle groups for three times a week improve the ability to balance and increase proprioception after balance training [24]. The unilateral, multilevel, static, and dynamic balance training program three times a week for 4 weeks also effectively improved joint proprioception and single-leg standing ability in subjects with unstable and nonimpaired ankles [49]. These studies showed that balance training is a useful method to improve balance in frail and nondisabled elderly people and confirmed our results, which show the effectiveness of balance training in patients with DN.
Having patients with DN focus on external visual biofeedback allows them to use more sensory strategies during training with an unstable platform. Some studies have confirmed the effectiveness of external visual biofeedback and have explained that focusing on the movement effect promotes an automatic mode of movement control [50-52]. An internal focus (active control of the body movements) is thought to disrupt automatic processes of normal movement control [52,53].

In our study, we found no significant differences between the pure effects of training, indicating that the balance performance of patients with DN improved in assessments with and without biofeedback as well as in assessments with open and closed eyes. Although patients with DN had similar improvement trends in balance control in the open and closed eyes conditions and the AP and ML directions, they improved significantly only in the open eyes ML-direction assessments, not under the closed eyes conditions.

Finally, the balance performance of patients with DN improved with a plan of balance training that paid attention to the disordered balance subclinical constraints and was intended to rehabilitate their somatosensory system at the ankle and hip levels. Furthermore, despite the improvements in all the BSS indexes, force platform analysis only improved in the open eyes-ML direction, a result that could be due to task-specific training.

In our assessments, we were limited to separate assessments of the somatosensory and vestibular systems. Thus, we could not estimate the unique role of each sensory modality in improving postural control. Moreover, we were limited in our assessment of clinical improvements. Furthermore, patients with BMI > 40 were excluded since we could not distinguish between postural adaptations due to DN and those due to obesity [54]. Furthermore, we had to eliminate the control group because neuropathy in each patient has its specific pattern. It was therefore difficult to match two groups of patients with DN with the same pattern of neuropathy, because the Valk score only summarized the levels of neuropathy. To solve this problem, we selected a timeseries quasi-experimental study as the optimal study method. In future work, a lower and more difficult level of BSS assessments may be useful for understanding the potential balance performance of patients with DN at those levels. 


\section{CONCLUSIONS}

Our findings support the hypothesis that training using sensory and reactive movement strategies with external visual feedback improves standing postural control in patients with DN by modifying the subclinical constraints that contribute to disordered balance. Thus, we suggest this approach for balance training of patients with DN to improve balance performance in this population.

\section{ACKNOWLEDGMENTS}

\author{
Author Contributions: \\ Study concept and design: H. Salsabili, F. Bahrpeyma. \\ Acquisition of data: H. Salsabili, B. Forogh. \\ Analysis and interpretation of data: H. Salsabili, S. Rajabali. \\ Drafting of manuscript: H. Salsabili. \\ Critical revision of manuscript for important intellectual content: \\ H. Salsabili, F. Bahrpeyma. \\ Statistical analysis: H. Salsabili. \\ Obtained funding: F. Bahrpeyma. \\ Administrative, technical, or material support: F. Bahrpeyma. \\ Study supervision: H. Salsabili, F. Bahrpeyma.
}

Financial Disclosures: The authors have declared that no competing interests exist.

Funding/Support: This material is the result of work supported with resources and use of facilities at Tarbiat Modares University of Medical Sciences, Tehran, Iran. This study was extracted from a Master of Physical therapy project and supported by the Tarbiat Modares University of Medical Sciences, Tehran, Iran.

Additional Contributions: We would also thank the department of Medical Physics of Shahid Beheshti University of Medical Sciences, Tehran, Iran, for their consultations.

Institutional Review: The ethical committees of Tarbiat Modares University approved the study protocol and informed consent that each patient signed prior to his or her participation. Patients were informed about the purposes of the study.

Participant Follow-Up: The authors do not plan to inform the participants of the publication of this study.

\section{REFERENCES}

1. Said G. Diabetic neuropathy-A review. Nat Clin Pract Neurol. 2007;3(6):331-40. [PMID: 17549059]

DOI:10.1038/ncpneuro0504

2. Boucher P, Teasdale N, Courtemanche R, Bard C, Fleury M. Postural stability in diabetic polyneuropathy. Diabetes Care. 1995;18(5):638-45. [PMID: 8586001]

3. Horak FB, Nashner LM, Diener HC. Postural strategies associated with somatosensory and vestibular loss. Exp
Brain Res. 1990;82(1):167-77. [PMID: 2257901]

DOI:10.1007/BF00230848

4. Bonnet C, Carello C, Turvey MT. Diabetes and postural stability: Review and hypotheses. J Mot Behav. 2009;41(2): 172-90. [PMID: 19201687] DOI:10.3200/JMBR.41.2.172-192

5. Horak FB, Dickestein R, Peterka RJ. Diabetic neuropathy and surface sway-referencing disrupt somatosensory information for postural stability in stance. Somatosens Mot Res. 2002;19(4):316-26. [PMID: 12590833] DOI:10.1080/0899022021000037782

6. Ahmmed AU, Mackenzie IJ. Posture changes in diabetes mellitus. J Laryngol Otol. 2003;117(5):358-64. [PMID: 12803785] DOI:10.1258/002221503321626393

7. Uccioli L, Giacomini PG, Monticone G, Magrini A, Durola L, Bruno E, Parisi L, Di Grilamo S, Menzinger G. Body sway in diabetic neuropathy. Diabetes Care. 1995; 18(3): 339-44. [PMID: 7555477$]$

8. Giacomini PG, Bruno E, Monticone G, Di Girolamo S, Magrini A, Parisi L, Menzinger G, Uccioli L. Postural rearrangement in IDDM patients with peripheral neuropathy. Diabetes Care. 1996;19(4):372-74. [PMID: 8729163] DOI:10.2337/diacare.19.4.372

9. Dickestein R, Shupert CL, Horak FB. Fingertip touch improves postural stability in patients with peripheral neuropathy. Gait Posture. 2001;14(3):238-47. [PMID: 11600327] DOI:10.1016/S0966-6362(01)00161-8

10. Lafond D, Corriveau H, Prince F. Postural control mechanisms during quiet standing in patients with diabetic sensory neuropathy. Diabetes Care. 2004;17(1):173-78.

[PMID: 14693985] DOI:10.2337/diacare.27.1.173

11. Horak FB, Hlavacka F. Somatosensory loss increases vestibulospinal sensitivity. J Neurophysiol. 2001;86(2):575-85. [PMID: 11495933]

12. Stergiou N. Innovative analyses of human movement. Champaign (IL): Human Kinetics; 2004.

13. Horak FB, Diener HC. Cerebellar control of postural scaling and central set in stance. J Neurophysiol. 1994;72(2): 479-93. [PMID: 7983513]

14. Morrison S, Colberg SR, Mariano M, Parson HK, Vinik AI. Balance training reduces falls risk in older individuals with type 2 diabetes. Diabetes Care. 2010;33(4):748-50.

[PMID: 20097781] DOI:10.2337/dc09-1699

15. Aly FA, Fawzy E, Ibrahim M, Mohamed A. Assessment of stability deficits in patients with Diabetic peripheral neuropathy. Bull Cairo Univ Fac Agric. 2007;12(1):31-42.

16. Yamamoto R, Kinoshita T, Momoki T, Arai T, Okamura A, Hirao K, Sekihara H. Postural sway and diabetic peripheral neuropathy. Diabetes Res Clin Pract. 2001;52(3):213-21. 
[PMID: 11323091]

DOI:10.1016/S0168-8227(01)00236-4

17. Corriveau H, Prince F, Hérbert R, Raîche M, Tessier D, Maheux P, Ardilouze JL. Evaluation of postural stability in elderly with diabetic neuropathy. Diabetes Care. 2000; 23(8):1187-91. [PMID: 10937520]

DOI:10.2337/diacare.23.8.1187

18. Oppenheim U, Kohen-Raz R, Alex D, Kohen-Raz A, Azarya M. Postural characteristics of diabetic neuropathy. Diabetes Care. 1999;22(2):328-33. [PMID: 10333953] DOI:10.2337/diacare.22.2.328

19. Macpherson JM, Fung J, Jacobs R. Postural orientation, equilibrium, and the spinal cord. Adv Neurol. 1997;72: 227-32. [PMID: 8993701]

20. Horak FB. Postural orientation and equilibrium: What do we need to know about neural control of balance to prevent falls? Age Aging. 2006;3 Suppl 2:ii7-ii11. [PMID: 16926210] DOI:10.1093/ageing/afl077

21. Shumway-Cook A, Woollacott M. Motor control: Translating research into clinical practice. 3rd ed. Philadelphia (PA): Lippincott Williams \& Wilkins; 2007. p. 257-99.

22. Allet L, Armand S, De Bie RA, Golay A, Monnin D, Aminian K, Staal JB, De Bruin ED. The gait and balance of patients with diabetes can be improved: A randomized controlled trial. Diabetologia. 2010;53(3):458-66.

[PMID: 19921145]

DOI:10.1007/s00125-009-1592-4

23. Tsang T, Orr R, Lam P, Comino EJ, Singh MF. Health benefits of Tai Chi for older patients with type 2 diabetes: The "Move It for Diabetes study"-A randomized controlled trial. Clin Interv Aging. 2007;2(3):429-39.

[PMID: 18044193]

24. Mattacola CG, Lloyd JW. Effects of a 6-week strength and proprioception training program on measures of dynamic balance: A single-case design. J Athl Train. 1997;32(2): 127-35. [PMID: 16558442]

25. Richardson JK, Sandman D, Vela S. A focused exercise regimen improves clinical measures of balance in patients with peripheral neuropathy. Arch Phys Med Rehabil. 2001; 82(2):205-9. [PMID: 11239311$]$

DOI:10.1053/apmr.2001.19742

26. Rose DJ, Clark S. Can the control of bodily orientation be significantly improved in a group of older adults with a history of falls? J Am Geriatr Soc. 2000;48(3):275-82. [PMID: 10733053]

27. Sihvonen S, Sipilä S, Taskinen S, Era P. Fall incidence in frail older women after individualized visual feedbackbased balance training. Gerontology. 2004;50(6):411-16. [PMID: 15477703] DOI:10.1159/000080180

28. Sihvonen SE, Sipilä S, Era PA. Changes in postural balance in frail elderly women during a 4-week visual feedback training: A randomized controlled trial. Gerontology. 2004; 50(2):87-95. [PMID: 14963375]

DOI:10.1159/000075559

29. Lindemann U, Rupp K, Muche R, Nikolaus T, Becker C. Improving balance by improving motor skills. Z Gerontol Geriatr. 2004;37(1):20-26. [PMID: 14991292]

DOI:10.1007/s00391-004-0206-5

30. Lee MY, Wong MK, Tang FT. Clinical evaluation of a new biofeedback standing balance training device. J Med Eng Technol. 1996;20(2):60-66. [PMID: 8836924] DOI:10.3109/03091909609008381

31. Hatzitaki V, Amiridis IG, Nikodelis T, Spiliopoulou S. Direction-induced effects of visually guided weight-shifting training on standing balance in the elderly. Gerontology. 2009; 55(2):145-52. [PMID: 18594127]

DOI:10.1159/000142386

32. Vinik AI, Strotmeyer ES, Nakave AA, Patel CV. Diabetic neuropathy in older adults. Clin Geriatr Med. 2008;24(3): 407-35. [PMID: 18672180$]$ DOI:10.1016/j.cger.2008.03.011

33. Tankisi H, Pugdahl K, Fuglsang-Fredriksen A, Johnsen B, De Carvalho M, Fawcett PR, Labarre-Vila A, Liguori R, Nix WA, Schofield IS; Esteem Project. Pathophysiology inferred from electrodiagnostic nerve tests and classification of polyneuropathies. Suggested guidelines. Clin Neurophysiol. 2005;116(7):1571-80. [PMID: 15907395]

DOI:10.1016/j.clinph.2005.04.003

34. Valk GD, Grootenhuis PA, Van Eijk JT, Bouter LM, Bertelsmann FW. Methods for assessing diabetic polyneuropathy: Validity and reproducibility of the measurement of sensory symptom severity and nerve function tests. Diabetes Res Clin Pract. 2000;47(2):87-95. [PMID: 10670907] DOI:10.1016/S0168-8227(99)00111-4

35. Arnold BL, Schmitz RJ. Examination of balance measures produced by the Biodex stability system. J Athl Train. 1998; 33(4):323-27. [PMID: 16558529]

36. Schmitz RJ, Arnold B. Intertester and intratester reliability of a dynamic balance protocol using the Biodex stability system. J Sport Rehabil. 1998;7(2):95-101.

37. Winter DA. Human balance and posture control during standing and walking. Gait Posture. 1995;3(4):193-214. DOI:10.1016/0966-6362(96)82849-9

38. Lin D, Seol H, Nussbaum MA, Madigan ML. Reliability of COP-based postural sway measures and age-related differences. Gait Posture. 2008;28(2):337-42. [PMID: 18316191] DOI:10.1016/j.gaitpost.2008.01.005

39. Balasubramaniam R, Wing AM. The dynamics of standing balance. Trends Cogn Sci. 2002;6(12):531-36.

[PMID: 12475714] DOI:10.1016/S1364-6613(02)02021-1 
40. Peterka RJ. Sensorimotor integration in human postural control. J Neurophysiol. 2002;88(3):1097-1118.

[PMID: 12205132]

41. Cohen J. Statistical power analysis for the behavioral sciences. 2d ed. Hillsdale (NJ): L. Erlbaum Associates; 1988. p. 19-66.

42. Nashner LM, McCollum G. The organization of human postural movements: A formal basis and experimental synthesis. Behav Brain Sci. 1985;8:135-50. DOI:10.1017/S0140525X00020008

43. Maki BE, McIlory WE. Postural control in the older adult. Clin Geriatr Med. 1996;12(4):635-58. [PMID: 8890108]

44. Kim BJ, Robinson CJ. Effects of diabetic neuropathy on body sway and slip perturbation detection in older population. Int J Occup Saf Ergon. 2006;12(3):241-54. [PMID: 16984784]

45. Maki BE, Holliday PJ, Topper AK. A prospective study of postural balance and risk of falling in an ambulatory and independent elderly population. J Gerontol. 1994;49(2): M72-84. [PMID: 8126355]

46. Nagy E, Feher-Kiss A, Barnai M, Domján-Preszner A, Angyan L, Horvath G. Postural control in elderly subjects participating in balances training. Eur J Appl Physiol. 2007; 100(1):97-104. [PMID: 17333243] DOI:10.1007/s00421-007-0407-X

47. Nardone A, Godi M, Artuso A, Schieppati M. Balance rehabilitation by moving platform and exercises in patients with neuropathy or vestibular deficit. Arch Phys Med Rehabil. 2010;91(12):1869-77. [PMID: 21112428]

DOI:10.1016/j.apmr.2010.09.011

48. Nardone A, Grasso M, Schieppati M. Balance control in peripheral neuropathy: Are patients equally unstable under static and dynamic conditions? Gait Posture. 2006;23(3): 364-73. [PMID: 15896962]

DOI:10.1016/j.gaitpost.2005.04.002

49. Rozzi SL, Lephart SM, Sterner R, Kuligowski L. Balance training for persons with functionally unstable ankles.
J Orthop Sports Phys Ther. 1999;29(8):478-86. [PMID: 10444738]

50. Wulf G, Shea C, Park JH. Attention and motor performance: Preference for and advantages of an external focus. Res Q Exerc Sport. 2001;72(4):335-44. [PMID: 11770783]

51. McNevin NH, Shea CH, Wulf G. Increasing the distance of an external focus of attention enhances learning. Psychol Res. 2003;67(1):22-29. [PMID: 12589447]

52. Wulf G, McNevin N, Shea CH. The automaticity of complex motor skill learning as a function of attentional focus. Q J Exp Psychol A. 2001;54(4):1143-54. [PMID: 11765737] DOI:10.1080/02724980143000118

53. Wulf G, Prinz W. Directing attention to movement effects enhances learning: A review. Psychon Bull Rev. 2001;8(4): 648-60. [PMID: 11848583] DOI:10.3758/BF03196201

54. Baszczyk JW, Cielinska-Swider J, Plewa M, ZahorskaMarkiewicz B, Markiewicz A. Effects of excessive body weight on postural control. J Biomech. 2009;42(9):1295-1300. [PMID: 19386313] DOI:10.1016/j.jbiomech.2009.03.006

Submitted for publication August 29, 2010. Accepted in revised form March 21, 2011.

This article and any supplementary material should be cited as follows:

Salsabili H, Bahrpeyma F, Forogh B, Rajabali S. Dynamic stability training improves standing balance control in neuropathic patients with type 2 diabetes. J Rehabil Res Dev. 2011;48(7):775-86.

DOI:10.1682/JRRD.2010.08.0160

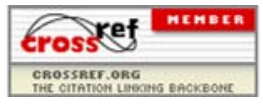

